\title{
Deep Multi-Scale Feature Learning for Defocus Blur Estimation
}

\author{
Ali Karaali, Naomi Harte, and Claudio R. Jung, Senior Member, IEEE
}

\begin{abstract}
This paper presents an edge-based defocus blur estimation method from a single defocused image. We first distinguish edges that lie at depth discontinuities (called depth edges, for which the blur estimate is ambiguous) from edges that lie at approximately constant depth regions (called pattern edges, for which the blur estimate is well-defined). Then, we estimate the defocus blur amount at pattern edges only, and explore an interpolation scheme based on guided filters that prevents data propagation across the detected depth edges to obtain a dense blur map with well-defined object boundaries. Both tasks (edge classification and blur estimation) are performed by deep convolutional neural networks (CNNs) that share weights to learn meaningful local features from multi-scale patches centered at edge locations. Experiments on naturally defocused images show that the proposed method presents qualitative and quantitative results that outperform state-of-the-art (SOTA) methods, with a good compromise between running time and accuracy.
\end{abstract}

Index Terms-Defocus blur estimation, multi-scale feature learning, deblurring

\section{INTRODUCTION}

When a camera captures real 3D scenes, the $2 \mathrm{D}$ projection on the image plane tends to present defocused regions due to the optical characteristics of lenses. Since the defocus blur amount depends on the distance of the captured objects to the focal plane, it generally varies from region to region in the image. This spatially varying blur is often represented by a defocus (blur) map that contains the size of the Circle of Confusion $(\mathrm{CoC})$, which is typically characterized as a disc or Gaussian and described by a radius/scale parameter $C_{\sigma}$ or $\sigma$.

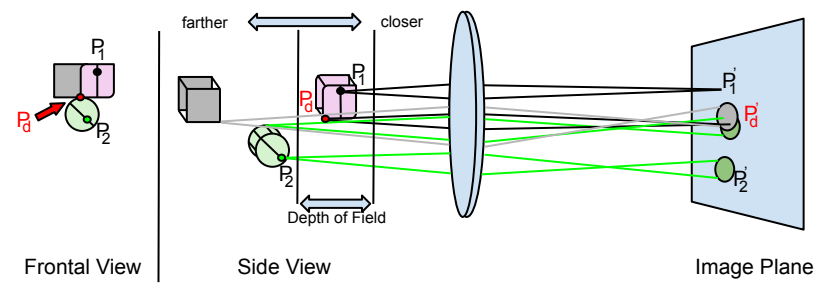

Fig. 1: Pictorial representation of how Circle of Confusions $(\mathrm{CoC})$ form from pattern and depth edges in a thin lens model.

Estimating the local defocus blur amount in images has many potential applications, including depth estimation [1], infocus object detection [2], [3], salience region detection [4], image retargeting [5], and non-blind deconvolution [6]. A

A. Karaali and N. Harte are with the School of Engineering, Trinity College Dublin. E-mail: (karaalia, nharte)@tcd.ie

C. Jung is with the Institute of Informatics, Federal University of Rio Grande do Sul. E-mail:crjung@inf.ufrgs.br typical categorization of existing defocus blur estimation methods is two-fold: edge-based and region-based methods. Edgebased methods use edge locations (which correspond to the high-frequency information and are more affected by blur) to estimate the local blur amount, then (optionally) propagate the defocus blur estimates from edge locations to the whole image. Region-based methods utilize local image patches and generally produce dense blur maps directly without any propagation scheme. In general, region-based methods are slower than edge-based methods since the defocus blur amount has to be estimated for each pixel location, while edge-based methods (initially) make estimates at only a fraction of the image pixels (i.e., the image edges) [7], [8].

The robustness of conventional defocus blur estimation methods [9], [10], [11] is highly dependent on the strength and/or isolation level of edges, making these methods prone to error since natural images tend to present complex edges with multiple intensity and isolation levels. Also, the vast majority of existing edge-based defocus blur estimation methods follow a similar blur approximation model as presented in Pentland's work [12], which relates the blur scale to the depth of an imaged 3D point based on a thin lens model. The main drawback of this formulation is that it is not valid for points that lie at depth discontinuities (e.g., boundaries between objects at different depth values), and the formulation should include a mixture of different blur parameters. Hence, assigning a single blur scale parameter to each edge point is ambiguous since the edge might be projected from a 3D point located at an object boundary with depth discontinuity. Fig. 1 shows a pictorial representation of this behavior based on a thin lens model. Rays coming from an edge point formed by the object boundary $\left(P_{d}\right)$ will be affected by other rays coming from other background (or foreground) objects, and the blur pattern will no longer be a simple circle. Instead, it will be formed by a combination of different circular patterns with different radii ( $P_{d}^{\prime}$ in Fig. 1). On the other hand, $P_{1}$ and $P_{2}$ are pattern edges related to object texture, presenting a relatively constant depth neighborhood and generating a well-defined $\mathrm{CoC}$ on the sensor plane.

Our main contribution in this paper is the introduction of a Convolutional Neural Network (CNN) feature learning approach that jointly tackles: i) the discrimination of depth edges (i.e., edges that lie at depth discontinuities) from pattern edges (i.e., edges that lie at relatively constant depth values); ii) multi-scale blur estimation for pattern edges that uses input patches with varying sizes to account for different local edge patterns. As an additional contribution, we adapt a fast edgeaware guided filter to propagate blur information estimated 
at pattern edge points to homogeneous regions, at the same time penalizing the propagation over depth edges. As will be discussed in the experimental results (Section IV), the final blur maps estimated by the proposed method for natural images yield competitive accuracy compared to all other SOTA methods in terms of the traditional error metric (MAE).

\section{RELATED WORK}

Defocus blur estimation methods can be divided into two main groups: edge- and region-based methods. Also, deep learning strategies have been developed for this task in recent years.

Edge-based methods generally follow a common strategy: estimating the unknown defocus blur amount along image edges, obtaining a sparse blur map, and then (optionally) propagating these blur estimates to the whole image via some interpolation/extrapolation methods to obtain a dense blur map (full blur map). Pentland [12], one of the pioneers of blur research, modeled a blurry edge by convolving a sharp edge with a Gaussian kernel. The standard deviation of the Gaussian kernel (i.e., the unknown blur scale) is then calculated using the intensity change rate along the edges. Elder and Zucker [13] proposed a simultaneous edge detection and blur estimation method. The method computes the blur scales measuring the zero-crossing of the third-order Gaussian derivatives along the gradient direction (using steerable filters). Both methods opted not to interpolate the blur estimates (e.g., estimated sparse blur maps only). Later on, Zhuo and Sim [9] used the gradient magnitude ratio between the original and a re-blurred version (of the original image) to estimate the local blur amount at edge points. A bilateral filter was employed to smooth the sparse blur map, and alpha Laplacian Matting [14] was chosen to propagate the estimated blur amounts to the rest of the image. The promising results of gradient magnitude usage (introduced in [9]) inspired many other researchers. For example, the use of more than one re-blurring parameter was proposed in [15], [16] to deal with noise, while a multi-scale approach was explored by Karaali and Jung [7] to deal with edge-scale ambiguity. On the other hand, Chen et al. [17] proposed a very fast full blur map estimation method, using the same sparse estimation scheme as in [9] with a novel propagation scheme. They first over-segmented the image using superpixels [18] and then computed an affinity matrix that measures the similarity between adjacent superpixels to assign a blur value to superpixels that do not overlap with image edges. Their method tends to produce piece-wise constant full blur maps.

Region-based methods typically examine local image patches in order to estimate the unknown blur amount and they mostly employ a post-regularization scheme to produce visually coherent results. In the pioneering work of Chakrabarti et al. [19], the blur identification problem (both defocus and motion) was tackled in the frequency domain by exploring the convolution theorem. They computed a likelihood function for a given candidate Point Spread Function (PSF), formulating a sub-band decomposition and Gaussian Scale Mixtures. This method later inspired Zhu et al. [20], who explored a continuous probability function to assess the unknown blur scale at each pixel location, analyzing the localized Fourier spectrum. Additionally, they incorporated color edge information and smoothness constraints to produce a coherent defocus blur map. Similar to [20], D'Andrès et al. [21] proposed the use of the localized Fourier spectrum, but they modeled the defocus blur estimation problem as image labeling. More specifically, they proposed labeling each image pixel with a discrete defocus blur scale using a machine learning method (regression tree fields - RTFs), which provides a global consistency in the estimated defocus map. Moreover, D'Andrès et al. in [21] created a naturally defocused image dataset with known (disk) defocus blur scales using a Lytro camera. Liu and colleagues [22] recently presented an extension of the RTF model by including edge information, which improved the results.

Deep learning research has shown promising results in many areas ranging from image classification [23], to superresolution [24] and image deblurring [25], to mention just a few applications. In recent years, interesting deep strategies have also been developed for defocus blur estimation. Zeng et al. [26] proposed a CNN architecture to learn meaningful local features in a superpixel level for blurry region estimation, and Zhang et al. [27] explored the blur "desirability" in terms of image quality (at three levels - Good, OK and Bad) using a huge manually labeled dataset, which is not currently publicly available. On the other hand, Park et al. [3] proposed combining hand-crafted features with deep features to boost the performance following a similar strategy to edge-based methods (e.g., sparse blur map estimation along edges followed by an interpolation). Lee et al. [28] recently presented an end-to-end $\mathrm{CNN}$ architecture for the defocus blur estimation problem, which uses an additional Domain Adaptation [29] technique to transfer features from naturally defocused images to synthetically defocused images. Tang and colleagues [30] also presented an end-to-end network based on a series of residual refinements, but focusing on blur detection (i.e., separating in-focus from out-of-focus regions). In particular, end-to-end methods (such as [28], [30]) can produce dense blur maps (as traditional region-based methods) while exploring tensor-based parallelism for fast execution, particularly when high-end GPUs are available. However, the performance tends to degrade as larger input images are used, as they might not fit entirely into the GPU memory.

In general, region-based approaches are costlier and more accurate than edge-based methods. In this paper, we present an edge-based method that outperforms existing edge- or regionbased methods in terms of accuracy. The core idea of our method is to use only pattern edge patches for defocus blur estimation to avoid $\mathrm{CoC}$ ambiguities at depth edge points. For this purpose, our solution explores deep architectures that first distinguish pattern from depth edges using multi-scale image patches (edge classification task), then estimate the blur values along pattern edges (blur estimation task), and finally generates a full blur map by using a fast propagation scheme that respects depth edges. To the best of our knowledge, the issue of $\mathrm{CoC}$ ambiguity at depth edge points has only been dealt with by Liu et al. [31], who model an edge point with two parameters (one parameter for each side of the edge). Although 

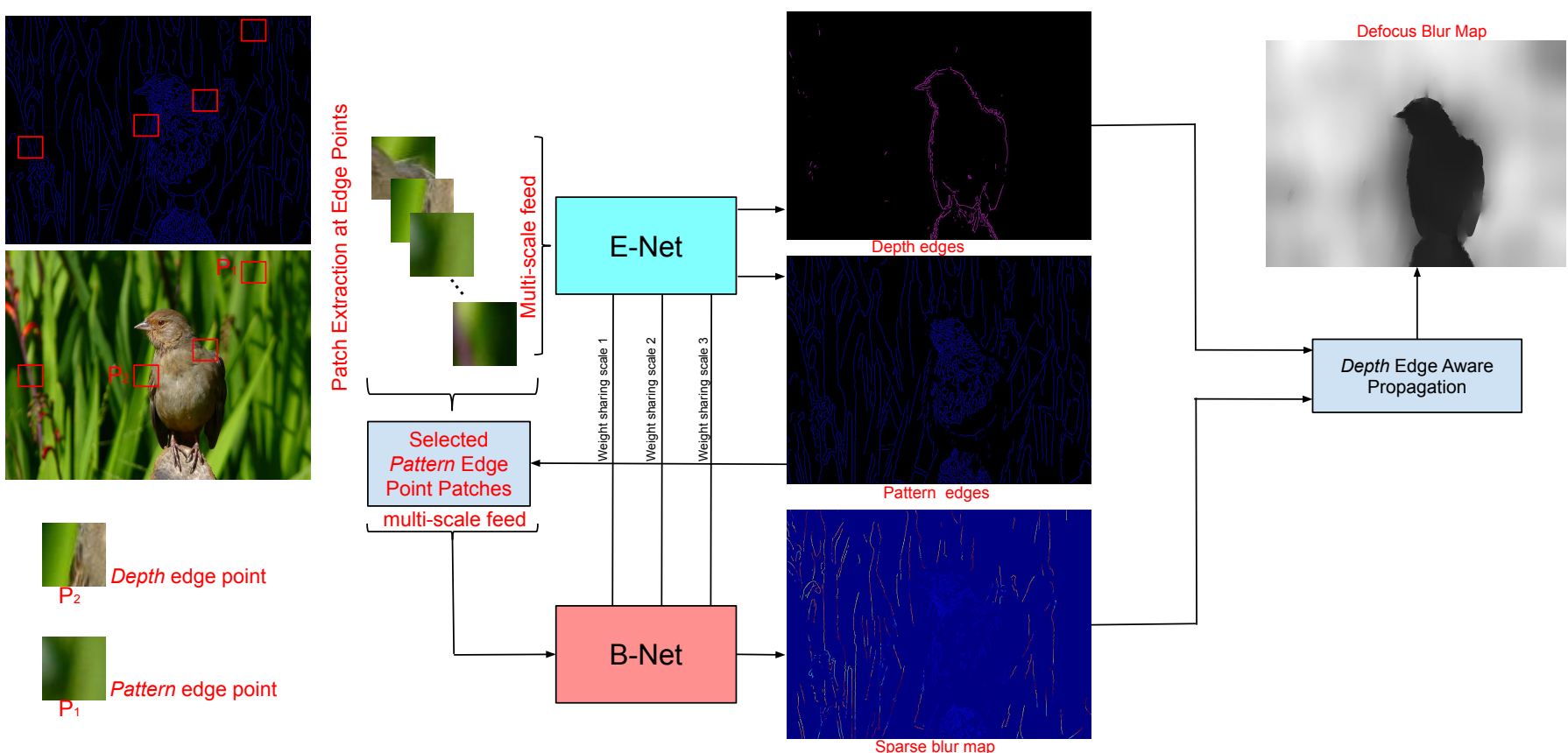

Fig. 2: Overview of our deep defocus blur estimation method.

their method produces visually plausible results, using a twosided blur model for depth edges is an oversimplification of the thin lens model since an edge point on a depth edge might contain a mixture of different Circle of Confusion (CoC) [32], [28]. Instead, we only use depth edges to prevent blur propagation across different objects.

\section{THE PROPOSED APPROACH}

Given a defocused input image $I^{B}$, our algorithm starts by computing an edge map of the input image. In this work, we used the well-known Canny detector [33], but any other edge detector can be used. Multi-scale image patches centered at the estimated edge locations are then fed to the first Convolutional Neural Network, called $\boldsymbol{E}-\boldsymbol{N E} \boldsymbol{T}$, which classifies an edge as depth- or pattern-related. The next step is to feed only patches related to pattern edges to another Convolutional Neural Network, called $\boldsymbol{B}-\boldsymbol{N E} \boldsymbol{T}$, which estimates the unknown defocus blur amount for a given edge point. Finally, a fast image-guided filter that propagates the sparse blur estimates to the whole image while penalizing propagation over depth edge points (which are related to object boundaries) is applied to obtain the final dense map. Fig. 2 presents an overview of our method.

Although the pattern vs. depth edge classification problem is related to the $3 \mathrm{D}$ geometry of the scene, our method does not explore structural information as in single-image stereo approaches, such as [34]. Instead, we explore local blur information caused by defocus, which typically occurs when shallow Depth-of-Field (DoF) cameras are used. As such, both tasks (edge classification and blur estimation) are expected to share low-level features, which suggests some kind of communication between the two networks. Since blur estimation is a consolidated problem with existing annotated datasets (unlike the proposed edge classification task), we hypothesize that the low-level features learned in $\boldsymbol{B}-\boldsymbol{N E T}$ can leverage the results of $\boldsymbol{E}$-NET . In fact, our initial tests explored one distinct network for each task (no weight sharing) and also a single network that branches off into the two tasks (full sharing of initial layers), but our partial weight sharing, as described next, presented the best results.

\section{A. Blur Estimation Network}

Our blur estimation network (B-NET) is fed with image patches centered at pattern edges. As noted in [35], determining an appropriate patch size is a key issue in order to avoid patch scale ambiguities. In the context of blur estimation, Park et al. [3] used an edge strength measure to determine suitable patch sizes, assuming that strong edges are likely to be less blurry than weak edges. Claiming that blurrier patches require more spatial information in the representation, they used larger patches for weak edges and smaller patches for strong edges (since their network uses fixed-input patches, a padding procedure is adopted for small patches). Despite the good results shown by the authors, we contend that edge strength does not necessarily correlate to blurriness. In fact, a sharp low-contrast edge can be detected as a weak edge. In this work, we do not explicitly select a desired input patch size, but instead we propose a multi-scale model to fuse information at different resolution levels.

$\boldsymbol{B}$-NET consists of two cascaded sub-networks: f1-NET (green shaded box in Fig. 3) and $b$-NET (yellow shaded box in Fig. 3). Sub-network $f 1-N E T$ receives three patches of different sizes $\left(P_{1}^{B}=41 \times 41, P_{2}^{B}=27 \times 27\right.$ and $\left.P_{3}^{B}=15 \times 15\right)$, which are extracted by centering the different-sized patches to the same edge location. These multi-scale patches, after a series of convolutional filters, Rectified Linear Units (ReLUs) and max pooling layers, are concatenated at the point where they reach the same spatial size (i.e. information at different resolution levels are fused), aiming to extract low-level blur information regarding the edges in a multi-scale way. It is 


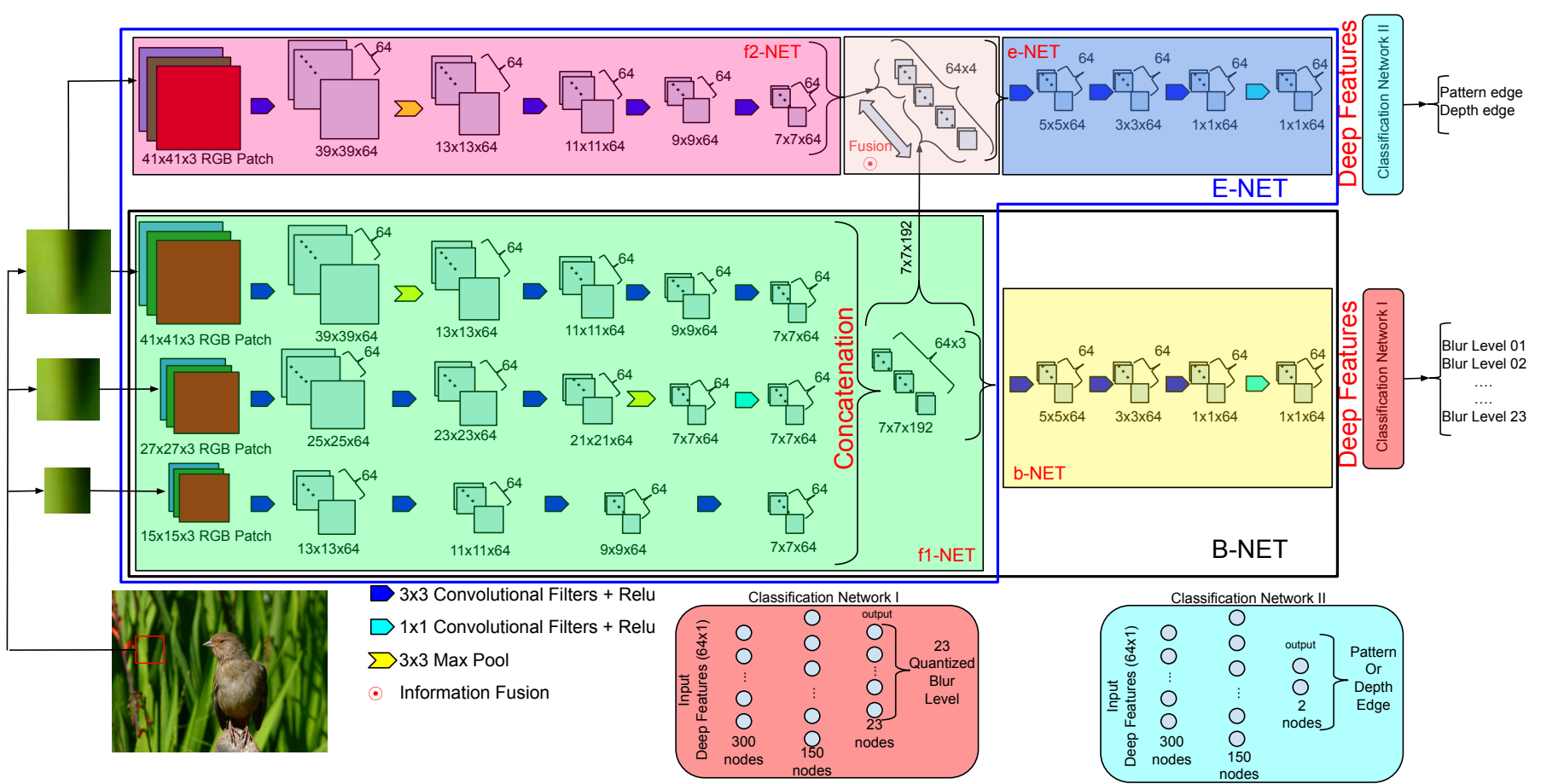

Fig. 3: Overview of the feature extraction networks for depth and pattern edge separation and defocus blur estimation.

important to note that we tried the same architecture using a single patch size (we tested different sizes), and the multiscale approach gave better results than any individual patch size.

The output of fl-NET is then fed to $b-N E T$, which consists of another sequence of convolutional filters with ReLU activation functions. The goal of $b-N E T$ is to extract deep features $f_{B}$ specialized to encode the blur level of the multiscale patches. These features $f_{B} \in \mathbb{R}^{64 \times 1}$ are then sent to a classification network called "Classification Network I", which consists of three fully connected layers. This network classifies the input feature vector $f_{B} \in \mathbb{R}^{64 \times 1}$ as one of the quantized blur levels (23 levels $\left.{ }^{1}\right)$ through two hidden layers (300 and 150 nodes of each) and one output layer with 23 nodes. ReLU activation functions are used in both hidden layers, and the softmax classifier is used in the last layer.

\section{B. Depth vs. Pattern Edge Classification}

Typical edge-based defocus blur estimation methods [9], [10], [17], [15] start by applying an edge detection scheme to the input image, and estimate a blur value for every edge pixel. However, as discussed in Section I, edges related to a depth discontinuity do not present a well-defined blur value, since they are affected by more than one CoC. In this work, we distinguish depth from pattern edges using a deep CNN called $\boldsymbol{E}$-NET, with an architecture as illustrated in the top of Fig. 3.

$\boldsymbol{E}-\boldsymbol{N E T}$ is a network designed to extract local deep features to distinguish pattern from depth edge points. Our hypothesis is that the low-level features extracted by fl-NET using multiscale patches encodes relevant information not only for blur estimation, but also for edge classification. As such, $\boldsymbol{E}-\boldsymbol{N E} \boldsymbol{T}$

${ }^{1}$ Please check Section IV-A for details inherits all the weights from $f 1-N E T)$, and it also includes a separate branch that is fed with a fixed-sized patch $\left(P_{1}^{E}=\right.$ $41 \times 41)$ that aims to extract features tailored to the edge classification problem. This branch is called sub-network $f 2$ NET and is shown in a purple shaded box in Fig. 3.

The outputs of fl-NET (low-level blur information) and $f 2-N E T$ (edge classification features) are then fused together when they reach the same spatial resolution (see "Information Fusion" in Fig. 3). Fused information is then sent to $e-N E T$, which consists of a set of convolutional layers with ReLU activation functions to extract deep features $f_{E}$ specialized for pattern and depth edge classification. Finally, these deep features $f_{E} \in \mathbb{R}^{64 \times 1}$ are sent to a classification network called "Classification Network II", which consists of 2 fully connected hidden layers of size 300 and 150 nodes each with ReLU activations functions, similar to the the classification layers of $\boldsymbol{B}-\boldsymbol{N E T}$. However, the output layer presents only two nodes (pattern or depth edge) with softmax activation.

It is also important to note that there is significatly more labeled data for blur estimation than edge classification (in fact, we had to label data for this task). Hence, fl-NET learns low-level blur features with a large amount of data, while $f 2-N E T$ learns specific low-level information for edge classification with a more modest amount of data.

\section{Full Blur Map}

The output of $\boldsymbol{B}$-NET is a sparse and quantized blur map computed only at pattern edges provided by $\boldsymbol{E}-\boldsymbol{N E T}$. As in most edge-based methods [9], [16], [31], [3], [7], a propagation scheme is used to obtain a full blur map. For this task, the majority of edge-based defocus blur estimation methods [9], [16], [31], [3] adopted the Laplacian-based colorization scheme [14]. However, this propagation scheme is time-consuming, and it respects the edges of the original blurry 
image $I^{B}$ even at pattern edges, causing visible artifacts at the full blur map even when depth (and blur) does not vary.

An alternative approach would be to use a cross-domain filter that acts on both the sparse blur map $I_{S}$ and the binary edge map $E$, assuming that $I_{S}(\boldsymbol{x})=0$ when $E(\boldsymbol{x})=0$. If function $\beta$ defines an adaptive weight for each pair of pixels $(\boldsymbol{x}, \boldsymbol{y})$, the interpolated blur map $B$ is given by

$$
B(\boldsymbol{x})=\frac{\sum_{\boldsymbol{y} \in \mathcal{N}_{\boldsymbol{x}}} \beta(\boldsymbol{x}, \boldsymbol{y}) I_{S}(\boldsymbol{y})}{\sum_{\boldsymbol{y} \in \mathcal{N}_{\boldsymbol{x}}} \beta(\boldsymbol{x}, \boldsymbol{y}) E(\boldsymbol{y})}=\frac{\mathcal{F}\left(I_{S}, I\right)}{\mathcal{F}(E, I)},
$$

where $\mathcal{N}_{\boldsymbol{x}}$ is the neighborhood around pixel $\boldsymbol{x}$ that specifies the data propagation region (and can be the whole image), and $\mathcal{F}(J, I)$ denotes the cross-domain filtering of image $J$ using image $I$ as the guidance (the selection of the filtering approach impacts the weights $\beta$ ). The normalization (denominator) ensures that the contribution of all valid values within the neighborhood $\mathcal{N}_{\boldsymbol{x}}$ adds up to one.

Edge-aware filters (such as the bilateral filter) consist of adaptive weights $\beta$ that try to prevent mixing information across image edges. In our problem, however, we can (and should) allow propagation across pattern edges, but want to prevent propagation across depth edges, since they typically separate objects at different depths (and hence, blur values). One edge-aware filter that allows a suitable adaptation for treating pattern and depth edges in a different way is the Domain Transform (DT) filter presented in [36]. The core idea of the DT filter is to perform 1D domain transformations such that samples considered "different" are spatially "pushed apart" from each other. With this transformation, a convolution kernel with a fixed size acts like an edge-preserving filter (similarly to the bilateral filter), and two-dimensional filtering is achieved by alternating filtering along rows and columns. For more details on the DT filter, the reader is directed to [36].

In this work, we propose a simple modification of the domain transformation that adds a penalty to depth edges. The proposed domain transformation function $c t$ (in the continuous domain) that measures the distance between two 1D points $u \leq w$ is given by

$$
c t(u, w)=\int_{u}^{w} 1+\Psi(x)+\frac{\sigma_{s}}{\sigma_{r}} \sum_{k=1}^{c}\left|I_{k}^{\prime}(x)\right| d x,
$$

where $I_{k}^{\prime}$ denotes the derivative of the $k$-th color channel of guide image $I$ (along a line or column), and $\sigma_{s}, \sigma_{r}$ are parameters that control the spatial and color range of the kernel, as defined in [36]. Note that the value of $\operatorname{ct}(u, w)$ progressively increases as does the spatial and color distances between points $u$ and $v$. Our modification to [36] is the introduction of the cost term $\Psi(x)$ given by

$$
\Psi(x)=\left\{\begin{array}{ll}
\psi, & \text { if } x \text { is a depth edge } \\
0, & \text { otherwise }
\end{array},\right.
$$

and $\psi$ is a constant that defines the penalty introduced by depth edges. Note that $1+\psi$ becomes a lower bound for the distance between any to points $u$ and $w$ separated by a depth edge. Hence, if $\psi$ is sufficiently large, blur propagation between these two points is virtually stopped. The domaintransformed signal is then convolved with a low-pass filter with variance $\sigma_{s}^{2}$ (very fast implementation if box filters are used).

Since fine textures and/or noise can block blur propagation across pattern edges, the input blurry image $I^{B}$ is first simplified by filtering with the edge-aware filter itself (i.e., $\left.\hat{I^{B}}=\mathcal{F}\left(I^{B}, I^{B}\right)\right)$, then is used as the guidance image in the propagation given by Eq. (2). Note that a similar strategy was employed in [7], but without considering depth edges.

In terms of complexity, $\boldsymbol{B}-\boldsymbol{N E T}$ and $\boldsymbol{E}-\boldsymbol{N E T}$ require, respectively, 123.46 and 149.16 MFLOPs. Since these networks are fed with edge-centered patches, the main bottleneck of the proposed method relates to the total number of edges. The interpolation step is very fast, since the DT-filter can be implemented in linear time [36].

\section{EXPERIMENTS}

\section{A. Data Preparation}

We use images from then ILSVRC [23], MS-COCO [37] and HKU-IS [38] datasets in order to train the proposed network architectures. Due to the lack of annotated datasets with blur data, we strongly rely on synthetic data.

Synthetic data for defocus blur estimation: in order to generate a dataset with known ground truth blur value (i.e. defocus blur amount is know for a given pixel) to train $\boldsymbol{B}$ $\boldsymbol{N E T}$, we first manually select sharp (all-in-focus) images that do not contain any visually detectable blurry pixels (250 from ILSVRC and 250 from MS-COCO), and then convolve each selected sharp image $I^{S}$ with a blur kernel. Although the actual kernel depends on the camera and lens system, a disk kernel models a perfect lens system with a circular aperture, and was shown to be a good approximation in experiments with real images conducted in [20]. Here, we used disk kernels $C_{R_{i}}$ to generate blurry images $I^{B}$ through

$$
I_{i}^{B}=I^{S} * C_{R_{i}}, i \in\{1,2, \cdots, S\},
$$

where $R_{i}$ denotes the blur level (i.e., the kernel size), and $S$ is the number of quantized blur values. Following [21], we set $S=23$, starting from $R_{1}=0.5$ and increasing up to $R_{23}=6$ with a step size of 0.25 . As noted in [22], the chosen range contemplates the expected blur amount in most image resolutions, except for extremely defocused regions in ultrahigh resolution images. Then, we extract approximately $5 \mathrm{M}$ patches from the edge points of synthetically blurred images, assuring that they are equally distributed for each blur level. Although blurring the whole image with a single spatiallyinvariant blur kernel is clearly an oversimplification since it does not impose any blur variations due to depth changes, this approach has generalized well to the blur estimation problem, especially in region-based methods [19], [20]. Furthermore, the separation of depth and pattern edges from each other assures that the proposed network will only be fed by patches that do not present strong depth variations.

Synthetic data for depth vs. pattern edge separation: to train $\boldsymbol{E}-\boldsymbol{N E T}$, which distinguishes depth edges from pattern edges, we need edge points that present different blurriness (i.e. depth) levels at different sides. Although a database with similar characteristics is reported in [27], it was not made 

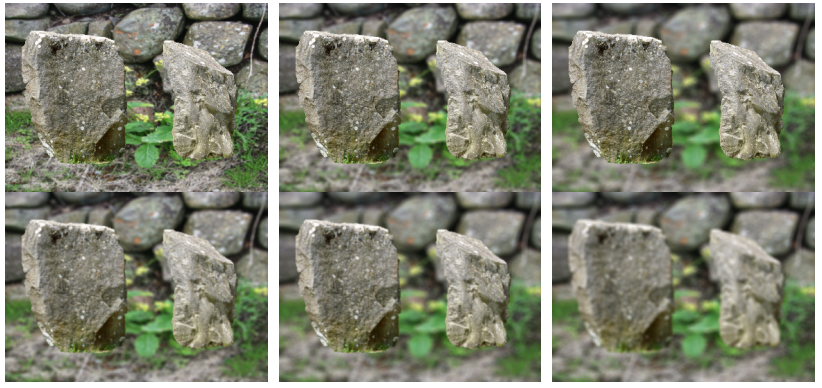

Fig. 4: Synthesized foreground-background images. Foreground blur level $R_{k_{1}}$, background blur level $R_{k_{2}}$. First row: $R_{k_{1}}=0$ and $R_{k_{2}}=1,3,5$, from left to right. Second row: $R_{k_{1}}=1$ and $R_{k_{2}}=3, R_{k_{1}}=1$ and $R_{k_{2}}=5$, and $R_{k_{1}}=3$, $R_{k_{2}}=5$ from left to right.

publicly available. Due to the lack of annotated data, we produced synthetic scenes $I^{F B}$ in a foreground-background manner using 200 salient regions as foreground objects from the HKU-IS [38] dataset, which provides images $S^{I}$ with binary masks $S^{B}$ indicating salient objects. We also use 100 images from ILSVRC [23] and 100 images from MSCOCO [37] datasets to compose the background of our dataset.

To generate our synthetic dataset, we first crop the salient object from the salient image $S^{I}$ using the provided binary mask $S^{B}$. Then, we blur the cropped region and its corresponding mask image with a disk blur kernel $C_{R_{k_{1}}}$, while simultaneously blurring a sharp (focused) image with a different disk blur kernel $C_{R_{k_{2}}}$ (with $R_{k_{1}}<R_{k_{2}}$ ), which will be the background image. Finally, we alpha-blend the blurry cropped image onto the blurry background image using the blurred binary mask as alpha values. We used four levels of blur scale for this task: $R_{1}=0$ for no blur, $R_{2}=1$ for low blur, $R_{3}=3$ is for medium blur and $R_{4}=5$ is for high blur.

For each salient image - background image pair, we synthesize $N=6$ synthetic foreground-background images $I_{n}^{F B}, n \in\{1,2, \ldots, N\}$, as illustrated in Fig. 4. Finally, we extract around $2 \mathrm{M}$ depth edge patches from the boundary of projected objects. Since using only synthetic images could overfit the network, we also manually labeled depth edges in real images, as illustrated in Fig. 5. More precisely, we labeled 100 real defocused images chosen from Flicker, from which $500 \mathrm{~K}$ depth edge patches were extracted. We also perform data augmentation by randomly rotating the images by $-90,-30$ 60,135 , or 180 degrees. Since noise did not seem to be an issue with the blurry images in our datasets, we did not add noise in the augmentation process.
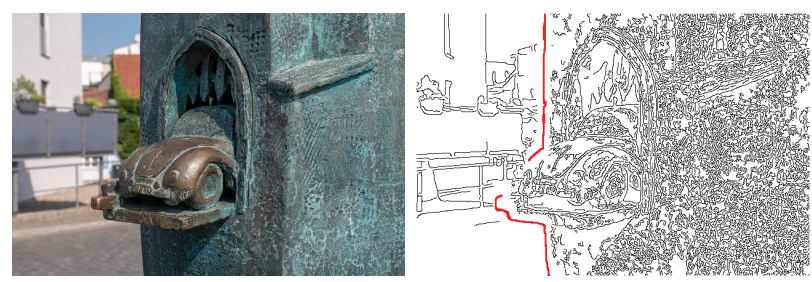

Fig. 5: Manually labeled depth edges from a defocused image in Flickr [39]
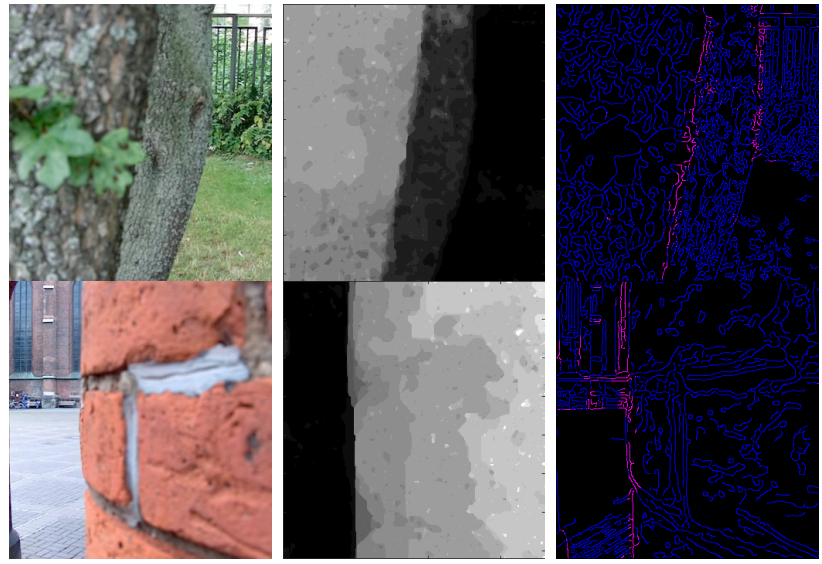

Fig. 6: Depth and pattern edge separation via $\boldsymbol{E}-\boldsymbol{N E T}$. From left to right (in pairs): original images (from [21]), ground truth blur map, and corresponding depth edges (red) and pattern edges (blue).

As for pattern edge patches, which is the other class label of $\boldsymbol{E}-\boldsymbol{N E T}$, we extract approximately $2.5 \mathrm{M}$ patches from 100 sharp (focused) images of ILSVRC [23] and 100 sharp (focused) images of MS-COCO [37] datasets, synthetically blurring them with different blur patterns such as uniform blur, gradually changing blur, and step-wise changing blur (with step size 0.25 to simulate small blur changes at the same depth layers).

\section{B. Model Training}

As described in Section III, although there is some weight sharing between $\boldsymbol{B}$ - $N \boldsymbol{E T}$ and $\boldsymbol{E}-\boldsymbol{N E T}$, the two networks are trained separately. Both models were implemented using Tensorflow with $80-20 \%$ train-validation data separation, with the softmax cross entropy as the loss function. We used the Adam [40] optimizer with a batch size of 256 .

We start by first training $\boldsymbol{B}$-NET, using an initial learning rate of $10^{-3}$ that is divided by 10 every 10 epochs, yielding convergence after 75 epochs. In a subsequent step we trained $\boldsymbol{E}-\boldsymbol{N E T}$ with the same initial learning rate, but divided by 10 at every 5 epochs instead of 10 . It is important to recall that during the training of $\boldsymbol{E}-\boldsymbol{N E T}$, the shared weights from $\boldsymbol{B}$ NET (called fl-NET, as shown in Fig. 3) are frozen, and the remaining weights of $\boldsymbol{E}-\boldsymbol{N E \boldsymbol { T }}$ are learned. With this strategy, the network converged after 30 epochs.

Implementation details: Regarding defocus blur interpolation, we used $\sigma_{r_{1}}=0.5$ and $\sigma_{s_{1}}=7$ to get the simplified image $\hat{I^{B}}$, and $\sigma_{r_{2}}=3.75$ and $\sigma_{s_{2}}=\min \{H, W\} / 8$ to propagate sparse blur estimates. $H$ and $W$ are the height and weight of the simplified defocus image $\hat{I}^{B}$ respectively. Before the simplification operation, the image pixels are normalized to the range $[0,1]$. Since the data interpolation has to be propagated to the whole image from edge pixels (which might be very sparse), we chose a large spatial kernel size $\sigma_{s_{2}}$. The range kernel $\sigma_{r_{2}}$ is also a relatively large value, chosen to increase pixel similarity and allow some propagation over pattern edges. For the penalization term of depth edges, we empirically set $\psi=100$ (which blocks propagation almost 
TABLE I: Quantitative evaluation of blur maps for the dataset provided in [21] in terms of Average MAE \pm Standard Deviation (STD) of Raw Blur, Average MAE of Relative Blur, Running Time and Implementation.

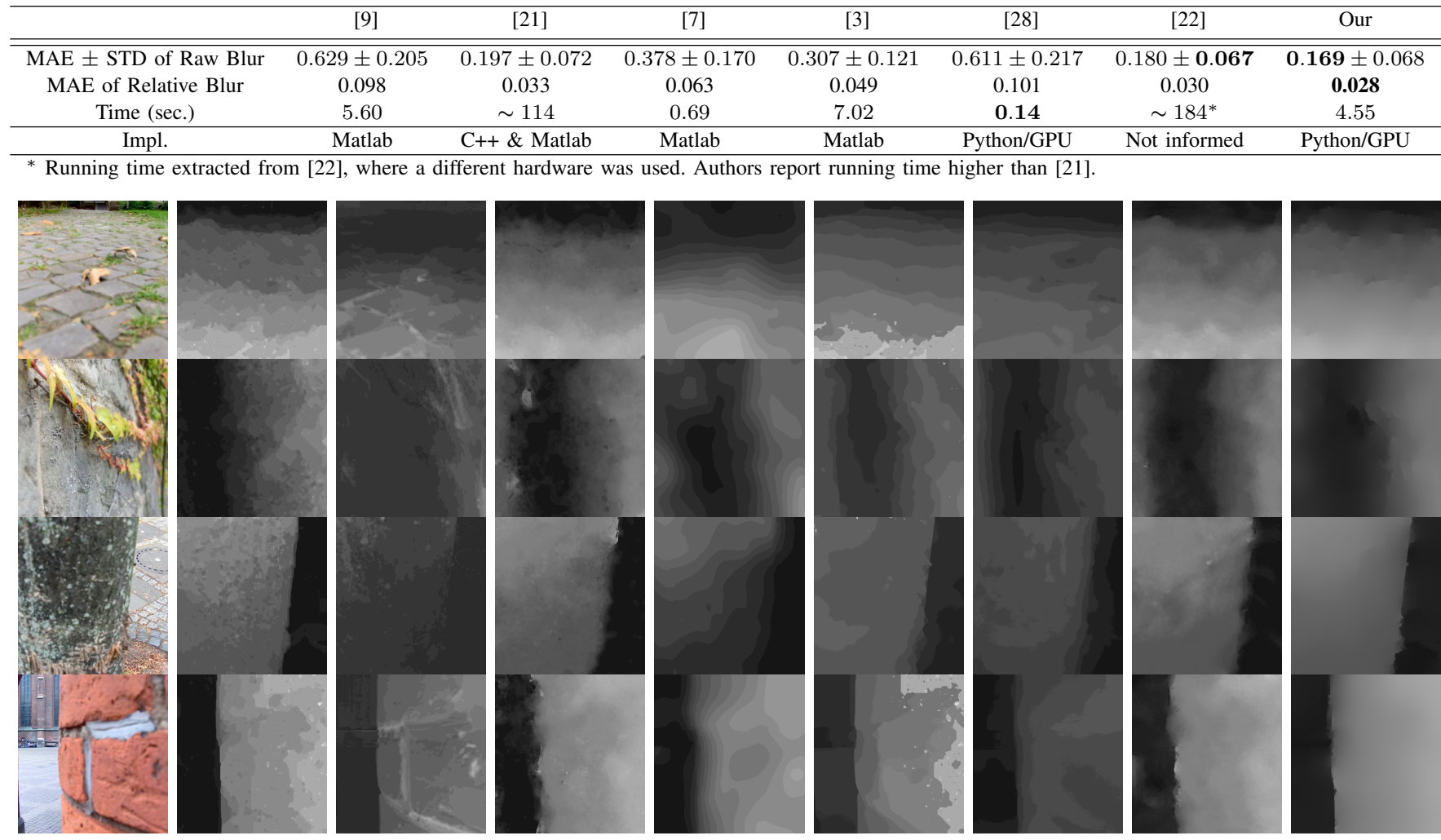

Fig. 7: Comparison of blur estimation algorithms using the dataset provided in [21]. From left to right: original image, ground truth, results of [9], [21], [7], [3], [28], [22] and the proposed method.

entirely) and used this default value in all experiments - larger values for $\psi$ show almost no difference from the default value.

\section{Experimental Validation}

In order to validate the proposed defocus blur estimation method, we use the dataset introduced in [21] that contains 22 real defocused images with resolution $360 \times 360$ captured by a Light Field camera, and it is (to our knowledge) the only dataset in the literature that provides the ground truth defocus blur values for each pixel. Although it also presents images corrupted by artificial noise, we restricted our analysis to the subset with natural image noise only since our model was not trained with artificial noise. We use the popular Mean Absolute Error (MAE) to quantitatively compare our defocus blur estimation approach with SOTA methods, and show edge map images with highlighted depth edges for qualitative evaluation of $\boldsymbol{E}$-NET on the same dataset.

We first illustrate examples of pattern vs. depth edge classification produced by $\boldsymbol{E}$-NET in Fig. 6. In the first blurry image, three different depth layers can be seen (from left to right), and $\boldsymbol{E}$-NET manages to distinguish most of the edge points that present depth discontinuities (abrupt blur change). In the second image, there is an abrupt depth transition from the red wall to the background, and $\boldsymbol{E}-\boldsymbol{N E \boldsymbol { T }}$ correctly labels these boundary points as depth edges, with a few false negatives. Note that edge classification is an intermediate step of our approach, and it will be evaluated implicitly by showing that it does improve the final goal (dense blur estimation), as will be shown next. Furthermore, the amount of data used to train $\boldsymbol{E}$-NET is rather limited, so we only split the data into train and validation (no test set). For the sake of illustration, the accuracy in the validation set was $88 \%$.

We computed the MAE of the raw blur values for each of the 22 images in the database and reported the average MAE and the standard deviation of the full blur maps obtained by the proposed method ${ }^{2}$ and competitive approaches in Table I. We also computed and reported the MAE of the relative blur values by re-scaling the raw blur values to $[0,1]$, as done in [28]. We can see that our method outperforms all the competitive approaches in both raw and relative blur ${ }^{3}$. It is slightly better than region-based methods [21], [22], which are considerably slower. Please note that as the method [28] produces a "relative blur map", they normalize the GT blur map by the maximum value to report their accuracy. For a fair comparison with the other approaches, we do the opposite: multiply their blur map by the maximum blur value to report the raw blur accuracy. Since the methods described in [9], [7], [3] model the blur with a Gaussian PSF, we re-scale their results from Gaussian scale to disk radii via the mapping function provided by [21]. Also, since the method presented in [3] is trained with a maximum Gaussian blur scale $\sigma_{g}=2.0$, we clipped the ground truth values to 2 when computing the

\footnotetext{
${ }^{2}$ Our code is available at github.com/alikaraali/DepthEdgeAwareBENet

${ }^{3}$ Although we used official implementation of [28] from github.com/ake/ DMENet, we obtained an average relative blur MAE slightly different from the value reported in their paper.
} 
TABLE II: Average PNSR \& SSIM for the deblurred images on the dataset provided in [21] using different blur maps. Best value shown in bold, second best in italic.

\begin{tabular}{cccccccccc}
\hline & Blurry Im. & GT Data & {$[9]$} & {$[21]$} & {$[7]$} & {$[3]$} & {$[28]$} & {$[22]$} & Our \\
\hline \hline PSNR/SSIM & $24.20 / 0.782$ & $\mathbf{2 7 . 6 9 / 0 . 8 8 5}$ & $23.96 / 0.786$ & $26.45 / 0.862$ & $25.72 / 0.833$ & $25.41 / 0.839$ & $24.70 / 0.801$ & $26.64 / 0.866$ & $27.00 / 0.870$ \\
Gain & N/A & $\mathbf{3 . 4 8 / 0 . 1 0 2}$ & $-0.24 / 0.004$ & $2.25 / 0.080$ & $1.51 / 0.050$ & $1.20 / 0.056$ & $0.49 / 0.018$. & $2.44 / 0.084$ & $2.80 / 0.088$ \\
\hline
\end{tabular}

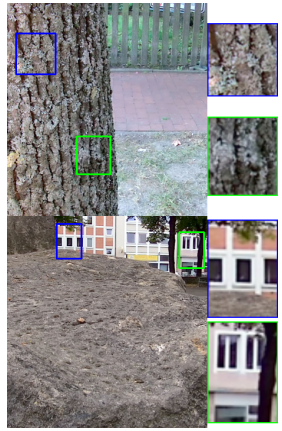

(a)

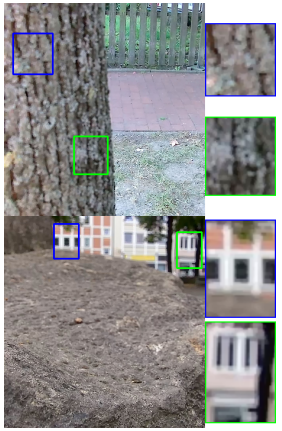

(b)

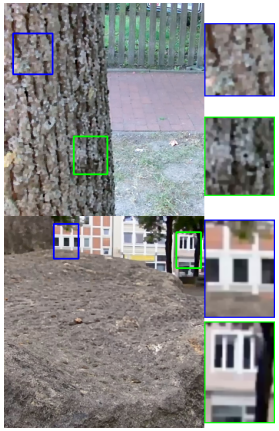

(c)

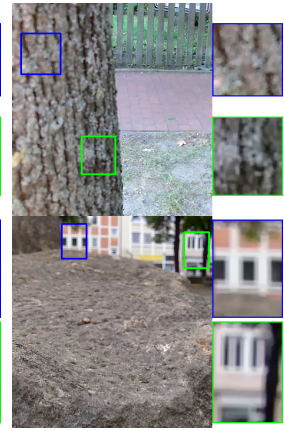

(d)

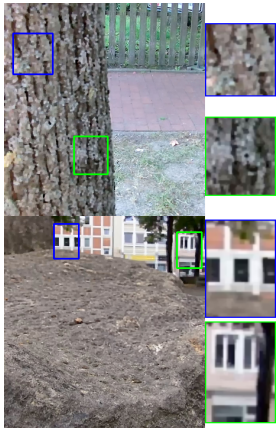

(e)

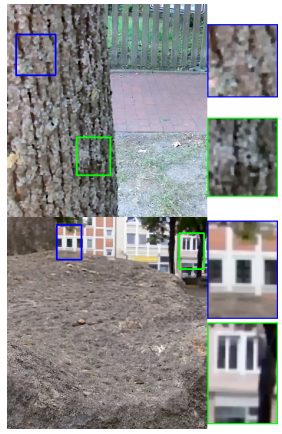

(f)

Fig. 8: Deblurring results using different blur maps as input. (a)-(b) sharp and blurry images. (c)-(f): results using the GT blur map, [28], [22] and the proposed method.

results of [3], which favored their results significantly. The average running times for all the analyzed methods considering all the images are also shown in Table I. Although [28] has the fastest execution speed, our method presents a very good compromise between MAE and running time when compared to other SOTA methods. The supplementary material provides a comprehensive study of the dataset.

Since an important application related to blur estimation is deblurring, we have also evaluated how well our method integrates in this task. Following [21], we used the combination of the methods proposed in [41] and [42] as a deblurring baseline approach, and used as input the ground truth data provided in [21], the defocus maps produced by SOTA approaches and by the proposed method. The average PSNR and SSIM values are summarized in Table II, showing that our method produced the highest average gain for both PSNR and SSIM, being inferior only to the deblurring results obtained with GT blur estimates. Fig. 8 shows a visual comparison of some deblurring results, highlighting regions with high structural or textural information. Our method presents visual results similar to those obtained with the ground truth blur map and the blur map produced with [22], while being much faster than [22]. A detailed analysis of this experiment, along with the visual results of all methods, is provided in the supplementary material.

Finally, we tested our method in the related task of defocus blur detection (DBD), which aims to find in-focus and outof-focus regions of a given image. For this task, we use the CUHK blur detection dataset [43], which contains 704 defocused images along with the corresponding binary blur maps as ground truth. Since the main focus of the proposed method is defocus blur estimation (i.e., finding the actual blur level at each pixel), DBD is performed by thresholding the estimated blur maps. More precisely, we used the same adaptive thresholding approach adopted in [3] and [28], which consists of defining a threshold

$$
\tau=\alpha v_{\max }+(1-\alpha) v_{\min },
$$

where $v_{\max }$ and $v_{\min }$ are the maximum and the minimum blur values of the estimated defocus blur map, and $\alpha$ is an empirically chosen parameter, which is set so as to maximize the accuracy of each method individually.

Fig. 9 shows the precision-recall curves for our approach and competitive methods, along with the corresponding accuracy (see legend of the figure). Since only a subset of 200 images was used to test the method presented in [28] (the remaining 504 were used to train it), all results refer to this smaller test subset for a fair comparison. It is important to mention that the proposed method, as well as [9], [7], and [3], does not use any images from CUHK dataset at any part of the algorithm design or training, while [28] uses images from CUHK dataset in the training phase for domain adaptation. As an illustration, the last two rows of Fig. 10 show some results produced by our method on images from the CUHK dataset (before thresholding), and more results are provided in the supplementary material.

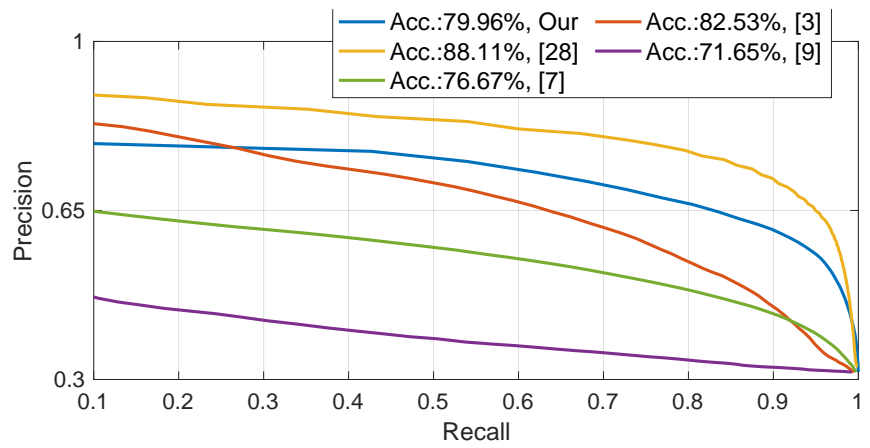

Fig. 9: Precision-recall curves and best accuracy for [9], [7], [3], [28] and the proposed method on the CUHK defocus detection dataset [43]. 

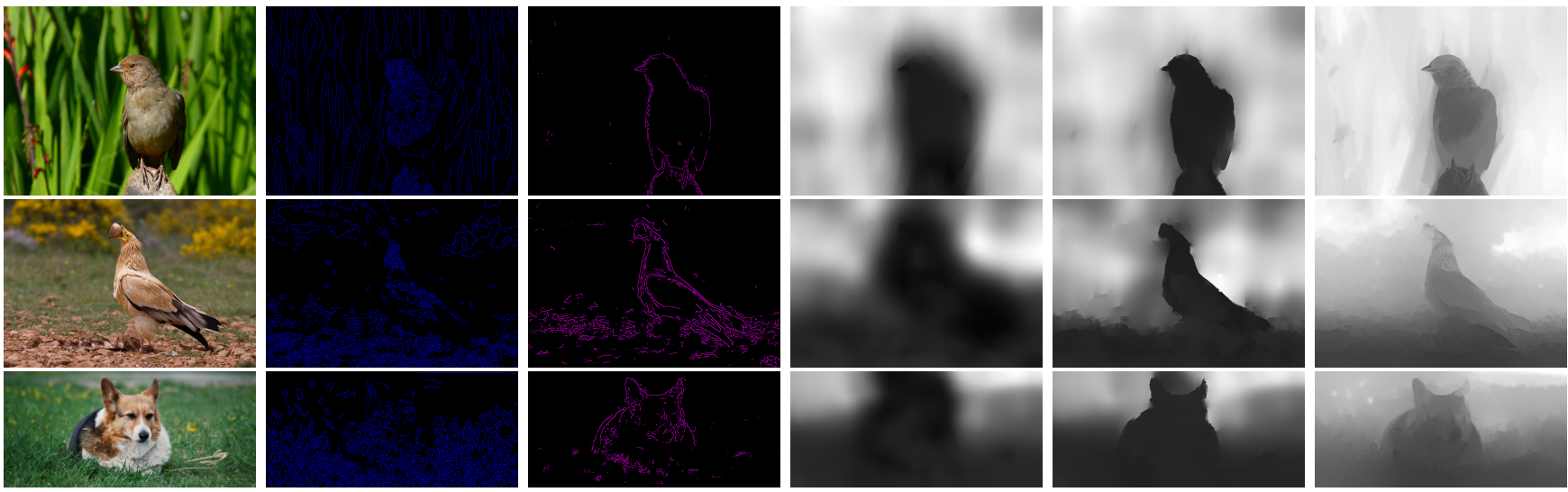

Fig. 10: Columns 1-3: original image, pattern edges, and depth edges. Columns 4-5: full blur maps via DT-Propagation without and with depth edge aware propagation. Last column: full blur map via Laplacian-based propagation

\section{Parameter Settings and Ablation Studies}

This section studies the effect of adding/removing some steps of the proposed approach, as well as the effect of changing some parameters. We start by noting that most of the existing edge-based defocus blur estimation methods adopt a post-processing scheme on the sparse blur estimates in order to smooth the results and get rid of outliers. For instance, Zhuo and Sim [9] used a joint bilateral filter with the original input image as the reference; Karaali and Jung [7] proposed a Connected Edge Filter (CEF) scheme that regularizes the blur estimates along connected edges; while Park et al. [3] proposed a probabilistic joint bilateral filter (PJBF), modifying the postprocessing approach used in [9] by including the confidence values of the blur estimates provided by their network. We tested all these three options in our sparse blur map, and although some of them yielded a small MAE drop on the sparse estimates, none provided any significant error changes in the final full blur maps. More precisely, the error (raw blur MAE) of sparse estimates decreased from 0.112 to 0.110 when used CEF post-filtering, but increased to 0.116 when used PJBF. On the other hand, the raw blur MAE of the corresponding full blur maps is 0.169 and 0.171 , respectively, which is equivalent to the results without any post-processing reported in Table $\mathrm{I}$.

Another important point to be addressed is the importance of distinguishing pattern from depth edges, and its relationship with the propagation scheme required to obtain the full blur map. In Table I, we showed that our average raw blur MAE for the natural blur dataset was 0.169 . We have also tested our propagation scheme without penalization across depth edges, and with the popular Laplacian-based colorization scheme [14] used in several competitive approaches [9], [16], [31], [3]. The average raw blur MAE for these two propagation schemes are 0.188 , and 0.288 , respectively. We show in Fig. 10 the result from $\boldsymbol{E}-\boldsymbol{N E T}$ and its impact on the dense blur map with and without propagation penalization across depth edges, along with the Laplacian-based scheme, for three images taken from [9] and [43]. Note that our scheme presents sharper object boundaries in the blur map for the in-focus objects. Also, note that not penalizing depth edges means setting $\psi=0$ in Eq. (2), so that changing $\psi$ from zero to 100 (default value) leads to increasing blockage of blur propagation across depth edges.

\section{CONCLUSIONS}

In this work, we have introduced a novel deep edge-based defocus blur estimation method. The main contributions of the proposed method were: the introduction of a network that distinguishes pattern and depth edges; use of only pattern edge points for blur estimation via another neural network architecture to avoid $\mathrm{CoC}$ ambiguity; and penalizing the propagation of sparse blur values at depth edge points to avoid mixing blur values from objects at different depths.

Quantitative results using the traditional MAE metric of raw and relative defocus blur estimates showed that the proposed blur estimation method produced more accurate results for natural blurry images than all the tested SOTA approaches. Furthermore, experiments involving image deblurring based on the estimated blur maps showed that our method outperformed competitive approaches in terms of the PSNR and SSIM of deblurred images. Finally, experiments conducted on the related task of defocus blur detection showed that the proposed method gave promising results with good cross-dataset generalization capabilities. We believe this is a remarkable achievement for an edge-based approach, particularly considering that we generated synthetic training data with a fixed kernel shape (disk) that is only an approximation of the actual blur kernel.

As future work, we plan to use different kernel shapes (e.g., disk and Gaussian) for generating training data with more variability aiming to further improve the generalization of the network. We also intend to develop another deep network that learns how to adequately propagate sparse blur information to the whole image given the original input color image.

\section{ACKNOWLEDGMENT}

This work was partly funded by the ADAPT Centre for Digital Content Technology, which is funded under the SFI Research Centres Programme (Grant 13/RC/2016) and is cofunded by the European Regional Development Fund, and also partly supported by Brazilian agencies CAPES (Finance Code 001) and CNPq. 


\section{REFERENCES}

[1] S. Gur and L. Wolf, "Single image depth estimation trained via depth from defocus cues," in The IEEE Conference on Computer Vision and Pattern Recognition (CVPR), June 2019.

[2] K. Zeng, Y. Wang, J. Mao, J. Liu, W. Peng, and N. Chen, "A local metric for defocus blur detection based on cnn feature learning," IEEE Transactions on Image Processing, vol. 28, no. 5, pp. 2107-2115, May 2019.

[3] J. Park, Y.-W. Tai, D. Cho, and I. S. Kweon, "A unified approach of multi-scale deep and hand-crafted features for defocus estimation," in Proc. of Computer Vision and Pattern Recognition (CVPR), 2017.

[4] P. Jiang, H. Ling, J. Yu, and J. Peng, "Salient region detection by ufo: Uniqueness, focusness and objectness," in Proceedings of the 2013 IEEE International Conference on Computer Vision, ser. ICCV ' 13. Washington, DC, USA: IEEE Computer Society, 2013, pp. 1976-1983.

[5] A. Karaali and C. R. Jung, "Image retargeting based on spatially varying defocus blur map," in 2016 IEEE International Conference on Image Processing (ICIP), Sep. 2016, pp. 2693-2697.

[6] H. E. Fortunato and M. M. Oliveira, "Fast high-quality non-blind deconvolution using sparse adaptive priors," The Visual Computer, vol. 30, no. 6, pp. 661-671, Jun 2014.

[7] A. Karaali and C. R. Jung, "Edge-based defocus blur estimation with adaptive scale selection," IEEE Transactions on Image Processing, vol. 27, no. 3, pp. 1126-1137, March 2018.

[8] A. Karaali, "Spatially varying defocus blur estimation and applications," PhD dissertation, Federal University of Rio Grande do Sul, 2017.

[9] S. Zhuo and T. Sim, "Defocus map estimation from a single image," Pattern Recognition, vol. 44, no. 9, pp. 1852 - 1858, 2011.

[10] W. Zhang and W. K. Cham, "Single-image refocusing and defocusing," IEEE Transactions on Image Processing, vol. 21, no. 2, pp. 873-882, Feb 2012.

[11] X. Zhang, R. Wang, X. Jiang, W. Wang, and W. Gao, "Spatially variant defocus blur map estimation and deblurring from a single image," Journal of Visual Communication and Image Representation, vol. 35, pp. $257-264,2016$.

[12] A. P. Pentland, "A new sense for depth of field," IEEE Transactions on Pattern Analysis and Machine Intelligence, vol. PAMI-9, no. 4, pp. 523-531, July 1987.

[13] J. H. Elder and S. W. Zucker, "Local scale control for edge detection and blur estimation," IEEE Transactions on Pattern Analysis and Machine Intelligence, vol. 20, pp. 699-716, 1998.

[14] A. Levin, D. Lischinski, and Y. Weiss, "A closed-form solution to natural image matting," IEEE Transactions on Pattern Analysis and Machine Intelligence, vol. 30, no. 2, pp. 228-242, 2008.

[15] Y. Cao, S. Fang, and Z. Wang, "Digital multi-focusing from a single photograph taken with an uncalibrated conventional camera," IEEE Transactions on Image Processing, vol. 22, no. 9, pp. 3703-3714, Sep. 2013.

[16] A. Karaali and C. R. Jung, "Adaptive scale selection for multiresolution defocus blur estimation," in Image Processing (ICIP), 2014 IEEE International Conference on, Oct 2014, pp. 4597-4601.

[17] D. J. Chen, H. T. Chen, and L. W. Chang, "Fast defocus map estimation," in 2016 IEEE International Conference on Image Processing (ICIP), Sept 2016, pp. 3962-3966.

[18] R. Achanta, A. Shaji, K. Smith, A. Lucchi, P. Fua, and S. Süsstrunk, "Slic superpixels compared to state-of-the-art superpixel methods," IEEE Transactions on Pattern Analysis and Machine Intelligence, vol. 34, no. 11, pp. 2274-2282, Nov 2012.

[19] A. Chakrabarti, T. Zickler, and W. T. Freeman, "Analyzing spatiallyvarying blur," 2013 IEEE Conference on Computer Vision and Pattern Recognition, vol. 0, pp. 2512-2519, 2010.

[20] X. Zhu, S. Cohen, S. Schiller, and P. Milanfar, "Estimating spatially varying defocus blur from a single image," Image Processing, IEEE Transactions on, vol. 22, no. 12, pp. 4879-4891, 2013.

[21] L. D'Andrès, J. Salvador, A. Kochale, and S. Süsstrunk, "Nonparametric blur map regression for depth of field extension," IEEE Transactions on Image Processing, vol. 25, no. 4, pp. 1660-1673, April 2016.

[22] S. Liu, Q. Liao, J.-H. Xue, and F. Zhou, "Defocus map estimation from a single image using improved likelihood feature and edge-based basis," Pattern Recognition, p. 107485, 2020.

[23] O. Russakovsky, J. Deng, H. Su, J. Krause, S. Satheesh, S. Ma, Z. Huang, A. Karpathy, A. Khosla, M. Bernstein, A. C. Berg, and L. Fei-Fei, "ImageNet Large Scale Visual Recognition Challenge," International Journal of Computer Vision (IJCV), vol. 115, no. 3, pp. 211-252, 2015
[24] S. Li, F. He, B. Du, L. Zhang, Y. Xu, and D. Tao, "Fast spatio-temporal residual network for video super-resolution," in The IEEE Conference on Computer Vision and Pattern Recognition (CVPR), June 2019.

[25] H. Gao, X. Tao, X. Shen, and J. Jia, "Dynamic scene deblurring with parameter selective sharing and nested skip connections," in The IEEE Conference on Computer Vision and Pattern Recognition (CVPR), June 2019.

[26] K. Zeng, Y. Wang, J. Mao, J. Liu, W. Peng, and N. Chen, "A local metric for defocus blur detection based on cnn feature learning," IEEE Transactions on Image Processing, vol. 28, no. 5, pp. 2107-2115, May 2019.

[27] S. Zhang, X. Shen, Z. Lin, R. Měch, J. P. Costeira, and J. M. F. Moura, "Learning to understand image blur," in The IEEE Conference on Computer Vision and Pattern Recognition (CVPR), June 2018.

[28] J. Lee, S. Lee, S. Cho, and S. Lee, "Deep defocus map estimation using domain adaptation," in The IEEE Conference on Computer Vision and Pattern Recognition (CVPR), June 2019.

[29] Y. Ganin and V. Lempitsky, "Unsupervised domain adaptation by backpropagation," in Proceedings of the 32Nd International Conference on International Conference on Machine Learning - Volume 37, ser. ICML'15. JMLR.org, 2015, pp. 1180-1189.

[30] C. Tang, X. Liu, X. Zhu, E. Zhu, K. Sun, P. Wang, L. Wang, and A. Zomaya, " $\mathrm{R}^{2} \mathrm{mrf}$ : Defocus blur detection via recurrently refining multi-scale residual features," in Proceedings of the AAAI Conference on Artificial Intelligence, vol. 34, no. 07, 2020, pp. 12 063-12070.

[31] S. Liu, F. Zhou, and Q. Liao, "Defocus map estimation from a single image based on two-parameter defocus model," IEEE Transactions on Image Processing, vol. 25, no. 12, pp. 5943-5956, Dec 2016.

[32] S. Lee, E. Eisemann, and H.-P. Seidel, "Real-time lens blur effects and focus control," ACM Trans. Graph., vol. 29, no. 4, pp. 65:1-65:7, Jul. 2010.

[33] J. Canny, "A computational approach to edge detection," IEEE Transactions on Pattern Analysis and Machine Intelligence, vol. PAMI-8, no. 6, pp. 679-698, Nov 1986.

[34] D. Eigen, C. Puhrsch, and R. Fergus, "Depth map prediction from a single image using a multi-scale deep network," in Advances in neural information processing systems, 2014, pp. 2366-2374.

[35] Q. Yan, L. Xu, J. Shi, and J. Jia, "Hierarchical saliency detection," in 2013 IEEE Conference on Computer Vision and Pattern Recognition, June 2013.

[36] E. S. L. Gastal and M. M. Oliveira, "Domain transform for edge-aware image and video processing," $A C M T O G$, vol. 30, no. 4, pp. 69:1-69:12, 2011, proceedings of SIGGRAPH 2011.

[37] T.-Y. Lin, M. Maire, S. Belongie, J. Hays, P. Perona, D. Ramanan, P. Dollár, and C. L. Zitnick, "Microsoft coco: Common objects in context," in Computer Vision - ECCV 2014, D. Fleet, T. Pajdla, B. Schiele, and T. Tuytelaars, Eds. Cham: Springer International Publishing, 2014, pp. $740-755$.

[38] Guanbin Li and Y. Yu, "Visual saliency based on multiscale deep features," in 2015 IEEE Conference on Computer Vision and Pattern Recognition (CVPR), June 2015, pp. 5455-5463.

[39] riesebusch, "Flickr," https://www.flickr.com/photos/127124365@N04/ 30785826128/in/dateposted/, 2019, [Online; accessed 2019].

[40] D. P. Kingma and J. Ba, "Adam: A method for stochastic optimization," in International Conference on Learning Representations (ICLR), 2014.

[41] D. Krishnan and R. Fergus, "Fast image deconvolution using hyperlaplacian priors," in Proceedings of the 22Nd International Conference on Neural Information Processing Systems, ser. NIPS'09, 2009, pp. 1033-1041.

[42] A. Levin, R. Fergus, F. Durand, and W. T. Freeman, "Image and depth from a conventional camera with a coded aperture," ACM transactions on graphics $(T O G)$, vol. 26 , no. 3, pp. 70-es, 2007.

[43] J. Shi, L. Xu, and J. Jia, "Discriminative blur detection features," in 2014 IEEE Conference on Computer Vision and Pattern Recognition, June 2014, pp. 2965-2972. 